\title{
The Lick AGN Monitoring Project 2011: Photometric Light Curves
}

\author{
Anna Pancoast ${ }^{1,2,18}$ (10), Andreas Skielboe ${ }^{3}$ (1) , Liuyi Pei $^{4}$, Vardha N. Bennert ${ }^{5}$ (1), David J. Sand ${ }^{6}$ (1), Aaron J. Barth ${ }^{4}$ (1), \\ Michael D. Joner ${ }^{7}$, Shawn Thorman ${ }^{4}$, Thomas Schmidt ${ }^{4}$, Tommaso Treu ${ }^{8}$ (1), Brendon J. Brewer ${ }^{9}$, Weidong Li ${ }^{10,19}$, \\ Tabitha Buehler ${ }^{7,11}$, C. David Laney ${ }^{7,12}$ (10), Gabriela Canalizo ${ }^{13}$, Alexei V. Filippenko ${ }^{10,14,20}$ (1), Jenny E. Greene ${ }^{15}$, \\ Matthew A. Malkan ${ }^{8}$ (1), Daniel Stern ${ }^{16}$ (1) , and Jong-Hak Woo ${ }^{17}$ (1) \\ ${ }^{1}$ Harvard-Smithsonian Center for Astrophysics, 60 Garden Street, Cambridge, MA 02138, USA; anna.pancoast@cfa.harvard.edu \\ ${ }^{2}$ Department of Physics, University of California, Santa Barbara, CA 93106, USA \\ ${ }^{3}$ Dark Cosmology Centre, Niels Bohr Institute, University of Copenhagen, Juliane Maries Vej 30, DK-2100 Copenhagen, Denmark \\ ${ }^{4}$ Department of Physics and Astronomy, 4129 Frederick Reines Hall, University of California, Irvine, CA 92697-4575, USA \\ ${ }^{5}$ Physics Department, California Polytechnic State University, San Luis Obispo, CA 93407, USA \\ ${ }^{6}$ Department of Astronomy and Steward Observatory, University of Arizona, 933 N Cherry Avenue, Tucson, AZ 85719, USA \\ ${ }^{7}$ Department of Physics and Astronomy, N283 ESC, Brigham Young University, Provo, UT 84602-4360, USA \\ ${ }^{8}$ Department of Physics and Astronomy, University of California, Los Angeles, CA 90095-1547, USA \\ ${ }^{9}$ Department of Statistics, The University of Auckland, Private Bag 92019, Auckland 1142, New Zealand \\ ${ }^{10}$ Department of Astronomy, University of California, Berkeley, CA 94720-3411, USA \\ ${ }^{11}$ Department of Physics and Astronomy, JFB 201, University of Utah, Salt Lake City, UT 84112, USA \\ ${ }^{12}$ Dept. of Physics and Astronomy, Western Kentucky University, 1906 College Heights Boulevard, Bowling Green, KY 42101, USA \\ ${ }^{13}$ Department of Physics and Astronomy, University of California, Riverside, CA 92521, USA \\ ${ }^{14}$ Miller Institute for Basic Research in Science, University of California, Berkeley, CA 94720, USA \\ ${ }^{15}$ Department of Astrophysical Sciences, Princeton University, Princeton, NJ 08544, USA \\ ${ }^{16}$ Jet Propulsion Laboratory, California Institute of Technology, 4800 Oak Grove Boulevard, Pasadena, CA 91109, USA \\ ${ }^{17}$ Astronomy Program, Department of Physics and Astronomy, Seoul National University, Seoul 151-742, Republic of Korea \\ Received 2018 August 31; revised 2018 November 27; accepted 2018 December 10; published 2019 January 25
}

\begin{abstract}
In Spring 2011, the Lick AGN Monitoring Project observed a sample of 15 bright, nearby Seyfert 1 galaxies in the $V$ band as part of a reverberation mapping campaign. The observations were taken at six ground-based telescopes, including the West Mountain Observatory $0.91 \mathrm{~m}$ telescope, the $0.76 \mathrm{~m}$ Katzman Automatic Imaging Telescope, $0.6 \mathrm{~m}$ Super-LOTIS at Kitt Peak, the Palomar 60 inch telescope, and the $2 \mathrm{~m}$ Faulkes telescopes North and South. The $V$-band light curves measure the continuum variability of our sample of Seyferts on an almost daily cadence for 2-3 months. We use image-subtraction software to isolate the variability of the Seyfert nucleus from the constant $V$-band flux of the host galaxy for the most promising targets, and we adopt standard aperture photometry techniques for the targets with smaller levels of variability. These $V$-band light curves will be used, with measurements of the broad emission line flux, to measure supermassive black hole masses and to constrain the geometry and dynamics of the broad-line region through dynamical modeling techniques.
\end{abstract}

Key words: galaxies: active - galaxies: nuclei - galaxies: Seyfert

Supporting material: machine-readable tables

\section{Introduction}

Variability in the luminosity of accreting supermassive black holes (BHs) in active galactic nuclei (AGNs) provides a method for measuring $\mathrm{BH}$ masses outside the local Universe using the reverberation mapping technique (Blandford \& McKee 1982; Peterson 1993; Peterson et al. 2004). Reverberation mapping works by measuring the distance of gas in the broad-line region (BLR) from the inner accretion disk that generates the optical and ultraviolet (UV) AGN continuum luminosity. Combining the size of the BLR with a measurement of the velocity of the BLR gas, determined from the width of a broad emission line, it is possible to obtain a dimensional $\mathrm{BH}$ mass measurement. Reverberation mapping constrains the size of the BLR by measuring the time lag between changes in the ionizing photon flux from the inner accretion disk and changes in the broad-line flux in the BLR due to reprocessing of ionizing photons. For the $>50$ AGNs with ground-based reverberation mapping data (Bentz \& Katz 2015), the optical

\footnotetext{
18 Einstein Fellow.

19 Deceased 2011 December 12.

${ }^{20}$ Miller Senior Fellow.
}

AGN continuum is used as a proxy for the true ionizing continuum.

Reverberation mapping is also used to constrain BLR structure, because the physical extent of the BLR is too small to resolve spatially using even the best current telescopes. Velocity-resolved lag measurements from reverberation mapping have found that the low-ionization BLR gas appears to have signatures consistent with nonradial motion around the BH in some objects (Peterson \& Wandel 1999; Bentz et al. 2009b; Denney et al. 2010; Barth et al. 2011a, 2011b; Grier et al. 2013; Du et al. 2016), although signatures of inflowing or outflowing BLR gas have also been found (Bentz et al. 2010b; Denney et al. 2010; Grier et al. 2013; Valenti et al. 2015; Du et al. 2016). Much of the analysis aimed at constraining the detailed BLR structure has focused on recovering the velocityresolved response function (e.g., Bentz et al. 2010b; Grier et al. 2013; Skielboe et al. 2015; Xiao et al. 2018), which shows the strength of the BLR response to continuum changes as a function of gas velocity and time lag $\tau$.

Another approach is to model the reverberation mapping data directly, assuming a simply parameterized model for the BLR (Brewer et al. 2011; Pancoast et al. 2012, 2014; 
Table 1

Details of the AGN Sample

\begin{tabular}{|c|c|c|c|c|c|}
\hline Object & $\alpha(\mathbf{J} 2000.0)$ & $\delta(\mathrm{J} 2000.0)$ & $z$ & $A_{V}(\mathrm{mag})$ & Alternate Name \\
\hline Mrk 40 & 112536.2 & +542257 & 0.0211 & 0.039 & Arp 151 \\
\hline Mrk 50 & 122324.1 & +024045 & 0.0234 & 0.044 & \\
\hline Mrk 141 & 101912.5 & +635803 & 0.0417 & 0.028 & \\
\hline Mrk 279 & 135303.4 & +691830 & 0.0305 & 0.044 & PG $1351+695$ \\
\hline Mrk 486 & 153638.3 & +543333 & 0.0389 & 0.040 & PG $1535+547$ \\
\hline Mrk 493 & 155909.6 & +350147 & 0.0313 & 0.068 & \\
\hline Mrk 504 & 170107.7 & +292425 & 0.0359 & 0.135 & PG $1659+294$ \\
\hline Mrk 704 & 091826.0 & +161819 & 0.0292 & 0.079 & \\
\hline Mrk 817 & 143622.1 & +584739 & 0.0315 & 0.019 & PG $1434+590$ \\
\hline Mrk 841 & 150401.2 & +102616 & 0.0364 & 0.082 & PG $1501+106$ \\
\hline Mrk 1392 & 150556.5 & +034226 & 0.0361 & 0.125 & \\
\hline Mrk 1511 & 153118.1 & +072728 & 0.0339 & 0.112 & NGC 5940 \\
\hline NGC 4593 & 123939.4 & -052039 & 0.0090 & 0.068 & Mrk 1330 \\
\hline PG 1310-108 & 131305.8 & -110742 & 0.0343 & 0.143 & II SZ 10 \\
\hline Zw 229-015 & 190525.9 & +422740 & 0.0279 & 0.198 & \\
\hline
\end{tabular}

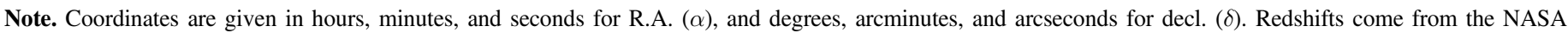
Extragalactic Database (NED). Galactic extinctions $\left(A_{V}\right)$ are taken from NED values from Schlafly \& Finkbeiner $(2011)$ with $R_{V}=3.1$.

Grier et al. 2017; Pancoast et al. 2018). Very high-quality reverberation mapping data are required to successfully apply these forms of analysis, motivating the need for long-duration reverberation mapping campaigns obtaining high signal-tonoise-ratio $(\mathrm{S} / \mathrm{N})$ spectra and simultaneous photometric monitoring of the AGN continuum. While the AGN continuum flux can be measured directly from the spectra, independent photometric monitoring provides higher-S/N light curves and does not suffer from the potential variability correlations between broad-line flux and continuum flux due to spectrophotometric calibration errors.

In 2008, the Lick AGN Monitoring Project (LAMP; Bentz et al. 2009b; Walsh et al. 2009) completed a reverberation mapping campaign with optical spectra taken at Lick Observatory and $B V$ imaging taken at a number of groundbased observatories. The LAMP 2008 sample was chosen to measure $\mathrm{BH}$ masses of $\sim 10^{6}-10^{7} M_{\odot}$, filling in the lower-mass regime of the $M_{\mathrm{BH}}-\sigma_{*}$ relation and providing a measurement of the average $f$ factor value (Woo et al. 2010), allowing for improved constraints on the BLR size-luminosity relation (Greene et al. 2010), and providing a recalibration of singleepoch AGN BH masses (Park et al. 2012). For the most variable object in the sample, Mrk 40 (Arp 151), the data allowed for reverberation mapping of multiple Balmer lines (Bentz et al. 2010b), constraints on the form of the velocityresolved response function using the maximum-entropy MEMEcho software (Bentz et al. 2010a), and, along with four other LAMP 2008 targets, dynamical modeling of the BLR to obtain independent measurements of the $\mathrm{BH}$ mass and $f$ factor (Brewer et al. 2011; Pancoast et al. 2014, 2018).

In order to obtain additional high-quality reverberation mapping data using spectra from Lick Observatory, the LAMP team undertook a second reverberation mapping campaign in the Spring of 2011, the Lick AGN Monitoring Project 2011. In addition to spectroscopy in the optical covering $\mathrm{H} \alpha$ through $\mathrm{H} \delta$ taken with the Kast spectrograph on the $3 \mathrm{~m}$ Shane telescope at Lick Observatory, $V$-band AGN continuum images were taken by six different telescopes. Preliminary results for LAMP 2011 focused on the most variable AGN in the sample, Mrk 50, including both a $\mathrm{BH}$ mass measurement using cross-correlation (Barth et al. 2011a) and a $\mathrm{BH}$ mass measurement from dynamical modeling of the BLR (Pancoast et al. 2012). Additional preliminary results for NGC 4593 and Mrk 1511 focused on reverberation of the Fe II broad-line complexes close to $\mathrm{H} \beta$ (Barth et al. 2013). Constraints on the BLR geometry, dynamics, and independent BH mass measurements have also been completed for eight AGNs, including reanalysis of Mrk 40 and Mrk 50 (Pancoast et al. 2018; Williams et al. 2018). Here, we measure the $V$-band AGN continuum light curves for the entire LAMP 2011 sample. The spectroscopic light curves are presented in a companion paper by Barth et al. (2015).

In this paper, we describe the analysis of $V$-band AGN continuum images for reverberation mapping. The resulting $V$-band light curves are measured using image-subtraction software for the higher-quality targets and traditional aperture photometry techniques for those showing less $V$-band or broad emission line variability. We align the light curves for each telescope by modeling the AGN continuum variability using Gaussian processes (GPs). Section 2 presents the LAMP 2011 sample of AGNs, and in Section 3 we describe the observations from the six telescopes. The data reduction and photometry are discussed in Section 4, including the Gaussian process model for aligning the different telescope light curves. In Section 5 we present the $V$-band continuum light curves and variability characteristics. We summarize our work in Section 6.

\section{Sample Selection}

The sample selection for this project was motivated by recent advances in measuring high-quality AGN light curves and analyzing velocity-resolved reverberation mapping data to obtain constraints on the geometry and dynamics of the BLR (Bentz et al. 2009b; Denney et al. 2010; Pancoast et al. 2011; Brewer et al. 2011). Since velocity-resolved reverberation mapping data require a high $\mathrm{S} / \mathrm{N}$ across the broad emission line profile, this sample focused on AGNs with $V \lesssim 17$ mag so that exposure times would not exceed 40 minutes. The LAMP 2011 sample includes 15 Seyfert 1 galaxies with $z<0.042$, listed in Table 1. These objects were chosen from the Sloan Digital Sky Survey (SDSS) Data Release 7 archive (Abazajian et al. 2009) and other AGN catalogs for strong broad $\mathrm{H} \beta$ emission. The time lag between the continuum and $\mathrm{H} \beta$ line flux was chosen to 
Table 2

Telescope Properties

\begin{tabular}{|c|c|c|c|c|c|}
\hline Telescope & $\begin{array}{l}\text { Mirror Diameter } \\
\qquad(\mathrm{m})\end{array}$ & FOV & $\begin{array}{c}\text { Scale } \\
(\text { ("/pixel) }\end{array}$ & $\begin{array}{c}\text { Gain } \\
\left(\mathrm{e}^{-} / \mathrm{ADU}\right)\end{array}$ & $\begin{array}{c}\text { Read Noise } \\
\qquad\left(\mathrm{e}^{-}\right)\end{array}$ \\
\hline WMO & 0.91 & $25 ! 2 \times 25 ! 2$ & 0.49 & 1.53 & 13.2 \\
\hline FTN & 2 & $10^{\prime} .5 \times 10^{\prime} .5$ & 0.304 & 8.09 & 11.31 \\
\hline KAIT & 0.76 & $6 ! 7 \times 6 ! 7$ & 0.8 & 4.5 & 12.0 \\
\hline Super-LOTIS & 0.6 & $17^{\prime} \times 17^{\prime}$ & 0.5 & 3.93 & 11.59 \\
\hline P60 & 1.5 & $12 ! 9 \times 12 ! 9$ & 0.378 & 2.2 & 5.72 \\
\hline
\end{tabular}

Note. FOV is field of view. The gain and read noise are parameters in the IRAF photometry procedure.

Table 3

Light-curve Statistics

\begin{tabular}{|c|c|c|c|c|c|}
\hline Object & $\begin{array}{c}\text { Exposure Time } \\
\text { (s) }\end{array}$ & $N$ & $\begin{array}{l}T_{\mathrm{med}} \\
\text { (days) }\end{array}$ & UT start & UT end \\
\hline Mrk 40 & $90-300$ & 91 & 0.94 & 2011 Mar 26 & $2011 \mathrm{Jul} 8$ \\
\hline Mrk 50 & $60-200$ & 170 & 0.61 & 2011 Jan 22 & 2011 Jun 25 \\
\hline Mrk 279 & $60-300$ & 64 & 1.02 & 2011 Feb 28 & 2011 Jun 15 \\
\hline Mrk 486 & $60-300$ & 95 & 0.95 & 2011 Mar 5 & 2011 Jun 20 \\
\hline Mrk 493 & $90-300$ & 79 & 0.86 & 2011 Apr 4 & 2011 Jun 19 \\
\hline Mrk 817 & $60-300$ & 100 & 0.95 & 2011 Mar 9 & $2011 \mathrm{Jul} 2$ \\
\hline Mrk 841 & $60-300$ & 89 & 0.96 & 2011 Mar 5 & $2011 \mathrm{Jul} 11$ \\
\hline Mrk 1392 & $60-300$ & 71 & 1.00 & 2011 Mar 5 & 2011 Jun 21 \\
\hline Mrk 1511 & $60-300$ & 85 & 0.91 & 2011 Mar 1 & 2011 Jun 15 \\
\hline NGC 4593 & $60-300$ & 75 & 0.94 & 2011 Mar 30 & 2011 Jun 21 \\
\hline PG $1310-108$ & $60-300$ & 63 & 1.04 & 2011 Mar 6 & 2011 Jun 15 \\
\hline
\end{tabular}

Note. Exposure time gives the range for an object from different telescopes. $N$ is the number of data points in the final light curve. $T_{\text {med }}$ is the median time between observations. UT start and end are the starting and ending dates for the light curves.

be $<25$ light days from the radius-luminosity relation by Bentz et al. (2009a) or previous reverberation mapping studies, ensuring that the campaign cadence would be smaller than the time lag for all objects and that the longest time lag could still be well constrained within the duration of the campaign. The full details of the LAMP 2011 sample selection are given by Barth et al. (2015).

\section{Observations}

We obtained $V$-band images of our sample of AGNs using six telescopes, as listed in Tables 2 and 3. The AGNs were monitored from early 2011 March through the middle of 2011 June. The exceptions were Mrk 50, for which spectroscopy was taken before the main observing campaign, and Mrk 817 and Zw 229-015, for which Spitzer Space Telescope data were taken during and after the campaign. Exposure times were generally $180-300 \mathrm{~s}$.

\subsection{West Mountain Observatory (WMO)}

The $0.91 \mathrm{~m}$ telescope at WMO is the largest of five telescopes operated by Brigham Young University. The telescope uses a $3056 \times 3056$ pixel Finger Lakes PL-09000 CCD with a scale of $0.49 /$ pixel and a field of view of $25 ! 2 \times 25 ! 2$. All AGNs in our sample were observed with WMO and photometric observations of each AGN were calibrated using WMO observations of Landolt fields (Landolt 1992), including G 163-51, PG 1407, SA110-503, and GL 163-5051.

\subsection{Faulkes Telescopes North and South}

The $2 \mathrm{~m}$ Faulkes Telescope North (FTN) and Faulkes Telescope South (FTS) are part of the Las Cumbres Observatory global telescope network (LCO; Brown et al. 2013). FTN is located at Haleakala Observatory, Hawaii, and FTS is located at Siding Spring Observatory, Australia, providing coverage of both the northern and southern hemispheres. For both telescopes we used the $4096 \times 4096$ pixel Spectral cameras with Fairchild CCD486 BI CCDs. The scale of the Spectral cameras is 0 ." $304 /$ pixel, providing a field of view of $10.5 \times 10.5$. We used FTN to observe Mrk 40, Mrk 141, Mrk 279, Mrk 486, Mrk 493, Mrk 504, Mrk 704, Mrk 817, and Zw 229-015, and FTS to observe Mrk 50, Mrk 841, Mrk 1392, Mrk 1511, NGC 4593, and PG 1310-108.

\subsection{Katzman Automatic Imaging Telescope (KAIT)}

The $0.76 \mathrm{~m} \mathrm{KAIT}$ is a robotic telescope at Lick Observatory (Filippenko et al. 2001). The telescope uses a $500 \times 500$ pixel Apogee Instruments Peltier cooled CCD with a scale of $0 . " 8 /$ pixel giving a field of view of $6 ! 7 \times 6 ! 7$. KAIT was used to 
observe all objects in the sample except Mrk 40, Mrk 486, Mrk 841, NGC 4593, and PG 1310-108.

\subsection{Super-LOTIS}

The $0.6 \mathrm{~m}$ Super-LOTIS telescope is a robotic telescope at the Steward Observatory, Kitt Peak. The telescope uses a $2048 \times 2048$ pixel Spectral Instruments 800 Series CCD Camera with a scale of $0.15 /$ pixel, giving a field of view of $17^{\prime} \times 17^{\prime}$. Super-LOTIS was used to observe all objects in the sample except $\mathrm{Zw}$ 229-015. Owing to a filter wheel problem, images obtained before March 26 were excluded from the lightcurve analysis.

\section{5. $P 60$}

The Palomar 60 inch (P60) telescope is a robotic telescope at the Palomar Observatory operated by the California Institute of Technology (Cenko et al. 2006). The telescope has a $2048 \times 2048$ pixel SITe CCD with a scale of 0 !" 378/pixel, giving a field of view of $12 ! 9 \times 12$ ! 9 . All objects in the sample were observed nightly.

\section{Data Reduction and Photometry}

Initial reduction of the data was performed by the automated pipeline for each telescope. The data then underwent additional reductions to ensure that the images from each telescope were bias-subtracted, and cosmic-ray-cleaned using the LA-COSMIC routine (van Dokkum 2001). Nine AGNs in the sample show significant $\mathrm{H} \beta$ variability, including Mrk 40, Mrk 50, Mrk 279, Mrk 504, Mrk 704, Mrk 1511, NGC 4593, PG 1310108, and Zw 229-015 (Barth et al. 2015). The preprocessed data for these nine AGNs were then analyzed using imagesubtraction software in order to isolate the variable AGN flux from the constant flux of the host galaxy. We performed photometry on the data with the constant flux sources removed using point-source IRAF photometry routines. The details of the photometry and image-subtraction process are described in Section 4.1. Since we created separate light curves for each telescope for a given AGN, we had to align the multiple light curves for each AGN as described in Section 4.2. A discussion of how the image-subtraction photometry flux uncertainties are calculated is given in Section 4.3 and the flux calibration of image-subtraction light curves in Section 4.5. For the remaining six AGNs without image-subtraction photometry, including Mrk 141, Mrk 486, Mrk 493, Mrk 817, Mrk 841, and Mrk 1392, the data were flat-fielded before we performed standard aperture photometry techniques as described in Section 4.4 .

\subsection{Image-subtraction Photometry}

The goal of the $V$-band imaging campaign is to measure differential photometry light curves for each AGN in the LAMP 2011 sample. Without a flux calibration, the differential photometry light curves have units of relative flux, which is sufficient for reverberation mapping analysis. What is crucial, however, is isolation of the variable AGN flux from the constant host-galaxy flux. The added constant host-galaxy flux has the effect of diluting the AGN variability, leading to less stringent constraints on the $\mathrm{BH}$ mass and lag measurements.

The traditional approach of creating differential photometry light curves for reverberation mapping involves photometric measurements on extended source images of the host galaxy plus the AGN. In this approach, a single aperture size is required for the photometry in each image of the light curve in order to minimize the effect of including a variable amount of host-galaxy light. However, variable seeing conditions over the course of the observing campaign can lead to more or less hostgalaxy light being inside the fixed-size aperture. Choosing the best aperture size to use is then an optimization problem of including as small a fraction of host-galaxy light as possible in order to not dilute the AGN signal, while using an aperture wide enough to not suffer from the effects of variable seeing conditions.

Image subtraction provides a natural solution to this problem by subtracting the constant flux sources in an image before photometry. We use image subtraction to create relative-flux light curves for the majority of AGNs in the LAMP 2011 sample. Once the variable AGN continuum has been isolated, we create $V$-band light curves using the point-source photometry of the AGN. This approach has also been taken by Fausnaugh et al. (2016), who used image subtraction to measure multiband continuum light curves for reverberation mapping of NGC 5548.

Applying image subtraction to a set of images is done by (1) astrometrically aligning the images, (2) creating a template image that has the best seeing and lowest background and is typically a combination of the best images, (3) creating individualized templates for each image by solving for the space-varying convolution kernel that matches the point-spread functions (PSFs) of the image and template, and (4) subtracting the template from each image. After subtracting the template from an image, only the variable components are left, including the AGN point source. While nonvariable comparison stars in the field of view are completely subtracted in this process, the amount by which their flux is subtracted is known and can be used to properly combine the different images to construct an AGN light curve.

We use the HOTPANTS image-subtraction package (Becker 2015), ${ }^{21}$ based on the algorithm by Alard (2000), and a version of the ISIS image-subtraction code (Alard \& Lupton 1998) modified by the High- $z$ Supernova Search Team (Tonry et al. 2003) and Weidong Li. For the highest quality images, the HOTPANTS and High-z software packages provide consistent results. However, for lower-quality images, one software package may provide a better image subtraction than the other, in which case only one software package is used for that light curve or individual image. Once the image subtraction has been completed, we perform point-source photometry on the image-subtracted images using the IRAF task PHOT in the DAOPHOT package to calculate the flux and corresponding uncertainty of the AGN and comparison stars. The photometry analysis consists of measuring the flux in a circular aperture centered on an object (AGN or comparison star) and then using a circular ring of flux outside the aperture to measure and subtract the background flux. After pointsource photometry, the light curve is in relative flux units.

We use a different template image for each telescope for a given AGN so that there are multiple light curves for each AGN, one from each telescope. The comparison stars for a given AGN field do not have to be the same for the different telescopes, making it easier to deal with fields of view of

\footnotetext{
${ }^{21}$ https://github.com/acbecker/hotpants
} 
varying size. In order to combine the different telescope light curves for an AGN, we model the light curves using GPs, as described in Section 4.2.

\subsection{Image Subtraction Light-curve Alignment}

After applying image subtraction and point-source photometry techniques to the $V$-band images, we are left with multiple relative-flux light curves for an $\mathrm{AGN}$, one for each telescope. While the standard aperture photometry procedure, described in Section 4.4, uses comparison star light curves to align the AGN light curves, this is not possible for the imagesubtraction procedure because the comparison stars used are not the same for each telescope. Instead, we align the different image-subtraction light curves for each AGN by modeling the light curve using GPs (MacKay 2003; Rasmussen \& Williams 2006), which has been found to be a good model for AGN variability on reverberation mapping timescales for large samples of AGNs (Kelly et al. 2009; Kozłowski et al. 2010; MacLeod et al. 2010; Zu et al. 2013). The model takes an arbitrary number of light curves and aligns them to a primary light curve. We use the WMO light curves as the primary light curves for our sample, because the WMO light curves are fluxcalibrated, as described in Section 4.5.

The model we use for the AGN light curves has a prior probability distribution for the vector of interpolated fluxes $\boldsymbol{y}$ in the light curve given by a multivariate Gaussian,

$$
\begin{aligned}
p(\boldsymbol{y} \mid \mu, C)= & \frac{1}{\sqrt{(2 \pi)^{n} \operatorname{det} \boldsymbol{C}}} \\
& \times \exp \left(-\frac{1}{2}(\boldsymbol{y}-\mu)^{T} \boldsymbol{C}^{-1}(\boldsymbol{y}-\mu)\right),
\end{aligned}
$$

where $\mu(t)$ is the mean function and $\boldsymbol{C}$ is the covariance matrix. The model is parameterized by four hyperparameters: $\mu$ (the long-term mean), $\sigma$ (the long-term standard deviation), $\tau$ (typical timescale of variations), and $\alpha$ (a smoothness parameter between 1 and 2), so that the mean function is a constant $\mu(t)=\mu$ and the covariance function between two points is given by

$$
C\left(t_{1}, t_{2}\right)=\sigma^{2} \exp \left[-\left(\frac{\left|t_{2}-t_{1}\right|}{\tau}\right)^{\alpha}\right] .
$$

We use GPs in the limit of a damped random walk (DRW) by setting $\alpha=1$, similar to past implementations for aligning reverberation mapping light curves by $\mathrm{Li}$ et al. (2014) and Fausnaugh et al. (2016). High-cadence Kepler data prefer a model with less variability at shorter timescales than the DRW model (e.g., Mushotzky et al. 2011; Kasliwal et al. 2015). However, Skielboe et al. (2015) found consistent results for GPs with and without $\alpha=1$ when calculating velocityresolved response functions for reverberation mapping data, suggesting that the analysis is not significantly affected by the DRW model's behavior at short timescales. The reason why our choice does not affect the alignment of the photometric light curves is that effectively GPs are used as a flexible means to interpolate the light curves accounting for uncertainties, and not to extract high-frequency information from the data.

We illustrate the light-curve alignment model using two light curves (true, complete, and noise-free), $y_{1}(t)$ and $y_{2}(t)$. There is a scaling $A$ and a shift $B$ that relates the two light curves, given by

$$
y_{2}(t)=A y_{1}(t)+B
$$

Suppose our knowledge of $y_{1}(t)$ is a Gaussian process (GP) with mean $\mu_{1}$ (a constant, for our purposes) and covariance function $C_{1}(\tau)$. By the linearity of Equation (3), our knowledge of $y_{2}(t)$ is also a GP, and indeed so is our joint knowledge of $y_{1}(t)$ and $y_{2}(t)$. The expectation value of $y_{2}(t)$ is

$$
\begin{gathered}
\mu_{2}=\left\langle y_{2}(t)\right\rangle=A\left\langle y_{1}(t)\right\rangle+B \\
=A \mu_{1}+B
\end{gathered}
$$

and the covariance function for $y_{2}(t)$ is

$$
\begin{gathered}
C_{2}(\tau)=\left\langle\left(y_{2}(t)-\mu_{2}\right)\left(y_{2}(t+\tau)-\mu_{2}\right)\right\rangle \\
=\left\langle\left(A y_{1}(t)+B-\left(A \mu_{1}+B\right)\right)\right. \\
\left.\times\left(A y_{1}(t+\tau)+B-\left(A \mu_{1}+B\right)\right)\right\rangle \\
=\left\langle\left(A y_{1}(t)-A \mu_{1}\right)\left(A y_{1}(t+\tau)-A \mu_{1}\right)\right\rangle \\
=A^{2} C_{1}(\tau) .
\end{gathered}
$$

We also need the "cross-terms," the covariance between $y_{1}\left(t_{1}\right)$ and $y_{2}\left(t_{2}\right)$. This is given by

$$
\begin{aligned}
& \operatorname{Cov}\left(y_{1}\left(t_{1}\right), y_{2}\left(t_{2}\right)\right)=\left\langle\left(y_{1}\left(t_{1}\right)-\mu_{1}\right)\left(y_{2}\left(t_{2}\right)-\mu_{2}\right)\right\rangle \\
&=\left\langle( y _ { 1 } ( t _ { 1 } ) - \mu _ { 1 } ) \left( A y_{1}\left(t_{2}\right)\right.\right. \\
&\left.\left.+B-\left(A \mu_{1}+B\right)\right)\right\rangle, \\
&= A\left\langle\left(y_{1}\left(t_{1}\right)-\mu_{1}\right)\right. \\
&\left.\times\left(y_{1}\left(t_{2}\right)-\mu_{1}\right)\right\rangle, \\
&=A C_{1}\left(t_{2}-t_{1}\right) .
\end{aligned}
$$

We use a Metropolis-Hastings Markov Chain Monte Carlo (MCMC) algorithm to explore the parameter space of $A, B$, and the parameters of the GP that include $\tau, \sigma, \alpha$, and $\mu$. The MCMC algorithm returns an inference on the parameter values in the form of the posterior probability density function (PDF); we use the mean values of the posterior PDFs for $A$ and $B$ for the final alignment of the light curves.

\subsection{Image Subtraction Light-curve Error Estimation}

The relative-flux uncertainties from image-subtraction analysis are measured by the IRAF photometry procedure PHOT in the DAOPHOT package. In many cases the flux uncertainties are very low for the high-quality images, owing to the imagesubtraction process isolating only the variable AGN point source from the extended host galaxy.

In order to obtain reasonable uncertainties on photometric measurements after image subtraction, there are two steps that must be taken. First, in order to properly account for the level of background flux in an image when using image subtraction and performing subsequent photometry, the background flux is not removed prior to performing image subtraction. Second, the aperture size for point-source photometry is only slightly larger than the typical PSF width of the images in the light curve. This second step ensures that the AGN variability is not diluted by the noise of the background in the image-subtraction-processed image.

Other conditions that affect the final flux uncertainties include the camera focus, observing conditions, and integration time of the exposure or number of exposures. These effects lead to each of the individual telescope light curves for an AGN 
having different typical flux uncertainties. We tested whether an example of the Super-LOTIS light curve having significantly larger uncertainties than the WMO and LCO data points was also true when carrying out photometry on the images before image subtraction. We used data for PG 1310-108 and performed extended source photometry on the AGN and three comparison stars for two images in the Super-LOTIS light curve for which the flux difference should be greatest based on higher-S/N data. We found that the difference in flux $(\Delta F)$ between these two points $F_{1}$ and $F_{2}$ divided by the uncertainty in this difference measurement $\left(\sigma_{\Delta F}=\sqrt{\sigma_{F_{1}}^{2}+\sigma_{F_{2}}^{2}}\right)$ was greater for image subtraction than for the extended source photometry. This shows that image subtraction is able to isolate the variable AGN flux with greater precision than extended source photometry. As a final test, we checked that the value $\Delta F / \sigma_{\Delta F}$ is conserved during realignment of each telescope light curve to that of WMO.

One source of systematic uncertainty that is not accounted for in the final light-curve fluxes is the uncertainty from aligning the light curves from different telescopes. As described in Section 4.2, we use an MCMC algorithm to constrain the values and uncertainties of the additive and multiplicative constants that align each telescope light curve to the WMO light curve. The uncertainty in the light-curve alignment is difficult to incorporate into the final light-curve values because it is a correlated error for all points in each telescope light curve. For an analysis that easily works with a sample of light curves instead of a single light curve, such as cross-correlation analysis, it is possible to use the posterior sample of light-curve alignments instead.

\subsection{Standard Aperture Photometry}

For objects that are comparatively less variable, we choose to use aperture photometry instead of image subtraction to construct the AGN light curves. This is because image subtraction is timeintensive and labor-intensive, while we use a fully automated aperture photometry routine that greatly simplifies the process of measuring light curves using heterogeneous data from multiple telescopes and instruments, as described by Pei et al. (2014). After the images are reduced, we register celestial coordinates onto the images using the Astrometry.net software (Lang et al. 2010). We then measure instrumental magnitudes for all AGNs and several comparison stars in each field by performing aperture photometry in IDL with an aperture radius of $4^{\prime \prime}$ and sky annulus radii of $10^{\prime \prime}-20^{\prime \prime}$. We choose comparison stars that have instrumental magnitudes similar to or slightly brighter than the AGN and we also take the average magnitude measurement for any night with multiple exposures.

First, separate AGN light curves are measured for each telescope. While the aperture photometry measures an uncertainty from photon-counting errors, uncertainties resulting from flat-field corrections, detector imperfections, and spatial variations of the PSF across the field of view are not included. We therefore calculate the excess variance of the comparison star light curves to obtain a more realistic estimate of the uncertainties:

$$
\sigma_{\mathrm{x}}^{2}=\frac{1}{N} \sum_{i=1}^{N}\left[\left(X_{i}-\mu\right)^{2}-\sigma_{i}^{2}\right],
$$

where $N$ is the number of points in the light curve, $\mu$ is the light curve's mean magnitude, the $X_{i}$ are the individual magnitude
Table 4

Image-subtraction Photometry

\begin{tabular}{lcccc}
\hline \hline Object & $\begin{array}{c}\text { HJD } \\
(-2,450,000)\end{array}$ & $\begin{array}{c}V \\
(\mathrm{mag})\end{array}$ & $\begin{array}{c}\text { error } \\
(\mathrm{mag})\end{array}$ & Telescope \\
\hline Mrk 40 & 5646.6401 & 15.706 & 0.030 & Super-LOTIS \\
Mrk 40 & 5648.6619 & 15.681 & 0.025 & Super-LOTIS \\
Mrk 40 & 5649.6621 & 15.690 & 0.030 & Super-LOTIS \\
Mrk 40 & 5650.6633 & 15.726 & 0.112 & Super-LOTIS \\
Mrk 40 & 5651.6652 & 15.643 & 0.028 & Super-LOTIS \\
\hline
\end{tabular}

(This table is available in its entirety in machine-readable form.)

Table 5

Standard Aperture Photometry

\begin{tabular}{lccll}
\hline \hline Object & $\begin{array}{c}\text { HJD } \\
(-2,450,000)\end{array}$ & $\begin{array}{c}V \\
(\mathrm{mag})\end{array}$ & $\begin{array}{c}\text { Error } \\
(\mathrm{mag})\end{array}$ & Telescope \\
\hline Mrk 141 & 5628.6207 & 15.394 & 0.011 & Super-LOTIS \\
Mrk 141 & 5629.8363 & 15.413 & 0.013 & Super-LOTIS \\
Mrk 141 & 5629.9040 & 15.372 & 0.008 & KAIT \\
Mrk 141 & 5629.9485 & 15.426 & 0.006 & LCO \\
Mrk 141 & 5630.8573 & 15.422 & 0.010 & Super-LOTIS \\
\hline
\end{tabular}

(This table is available in its entirety in machine-readable form.)

values in the light curve, and the $\sigma_{i}$ are the corresponding magnitude uncertainties. The final AGN light-curve uncertainties for each telescope are calculated by adding the average scatter from the excess variance in quadrature with the photoncounting errors from the photometry. Finally, the AGN light curves for each telescope are aligned to the light curve with the highest $\mathrm{S} / \mathrm{N}$, WMO, by matching the average value of each comparison star light curve.

\subsection{Light-curve Flux Calibration}

The final step for creating AGN light curves using either image subtraction or standard aperture photometry is to flux calibrate the light curves. For the light curves with standard aperture photometry in magnitudes, we flux calibrate the comparison star light curves with Landolt standard stars (Landolt 1992) observed on the same night with WMO, with comparison-star magnitudes published for AGN fields (Doroshenko et al. 2005a, 2005b), and from the APASS Database. ${ }^{22}$ We then apply the average difference between the instrumental and calibrated magnitudes calculated for 3-5 comparison stars to the AGN light curve. The uncertainty in the flux calibration is below $0.1 \mathrm{mag}$, resulting from the APASS flux accuracy.

For consistency, we apply the same procedure to both image subtraction and standard aperture photometry light curves by performing standard aperture photometry on the AGN with image-subtraction light curves. The image-subtraction light curves are then aligned to the flux-calibrated aperture photometry light curves using the same procedure as described in Section 4.2. The flux-calibrated image-subtraction light curves carry the same general uncertainty of $0.1 \mathrm{mag}$ in the overall flux calibration.

\footnotetext{
${ }^{22}$ https://www.aavso.org/apass
} 


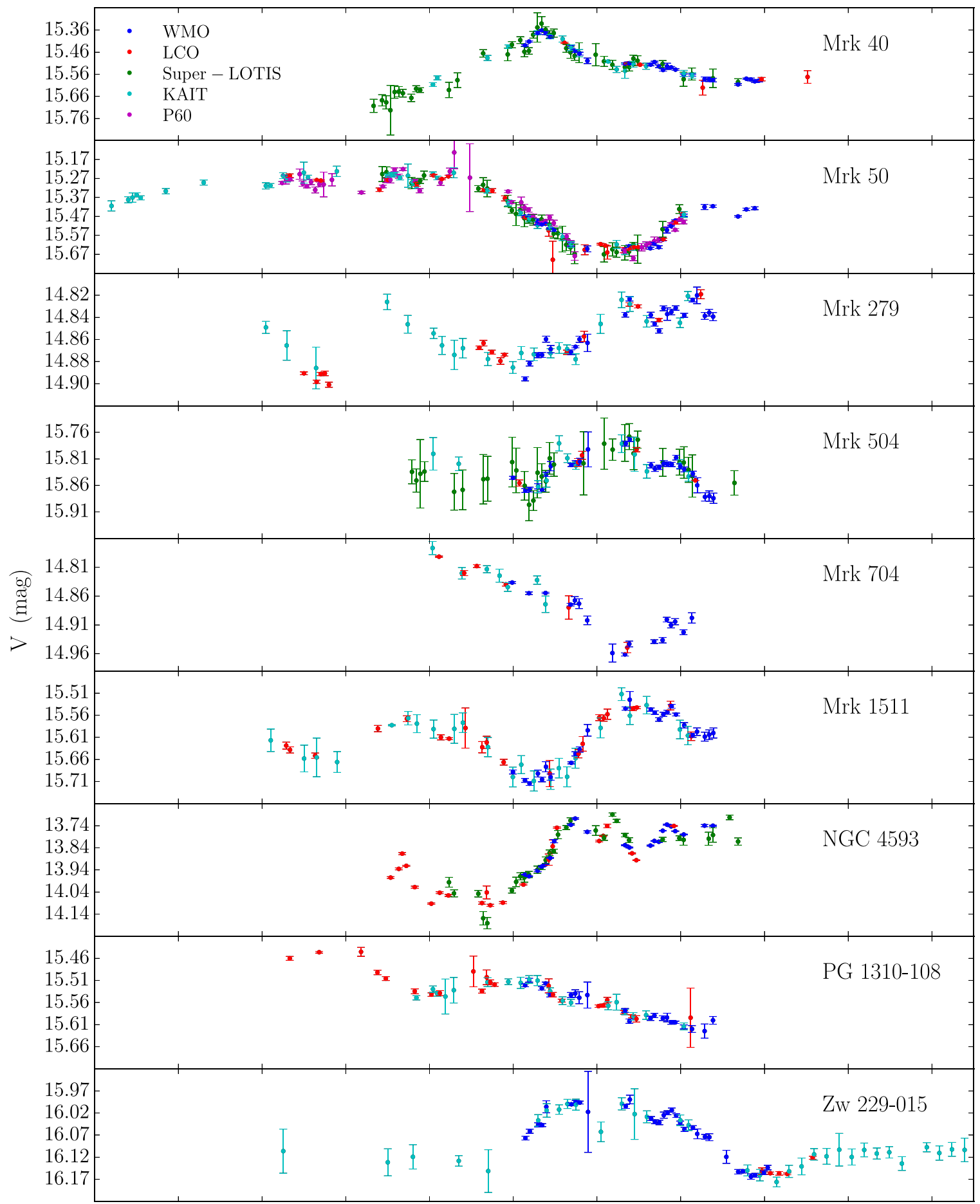

HJD - 2450000

Figure 1. Image subtraction light curves for LAMP 2011. The different colors correspond to data from different telescopes, as indicated in the legend. LCO data consist of either FTN or FTS. Mrk 50 has additional monitoring before the beginning of the main LAMP 2011 campaign and Zw 229-015 has additional monitoring at the end of the main campaign.

\section{Results}

The AGN $V$-band continuum light curves from image subtraction and standard aperture photometry are given in Tables 4 and 5 and shown in Figures 1 and 2, respectively. The light curves for each telescope are indicated with different colors in Figures 1 and 2 to show the range in data quality. Generally, the WMO and FTN/FTS (labeled as LCO) light curves are of the highest quality, but suffer from uneven or sparse sampling in the case of FTN/FTS, or a delay in the start of observations for WMO with respect to the beginning of the monitoring campaign. The KAIT and Super-LOTIS data are required to fill in many gaps, especially before the start of WMO monitoring. On the other hand, the P60 data suffer from rows of bad pixels, making it challenging to find comparison 


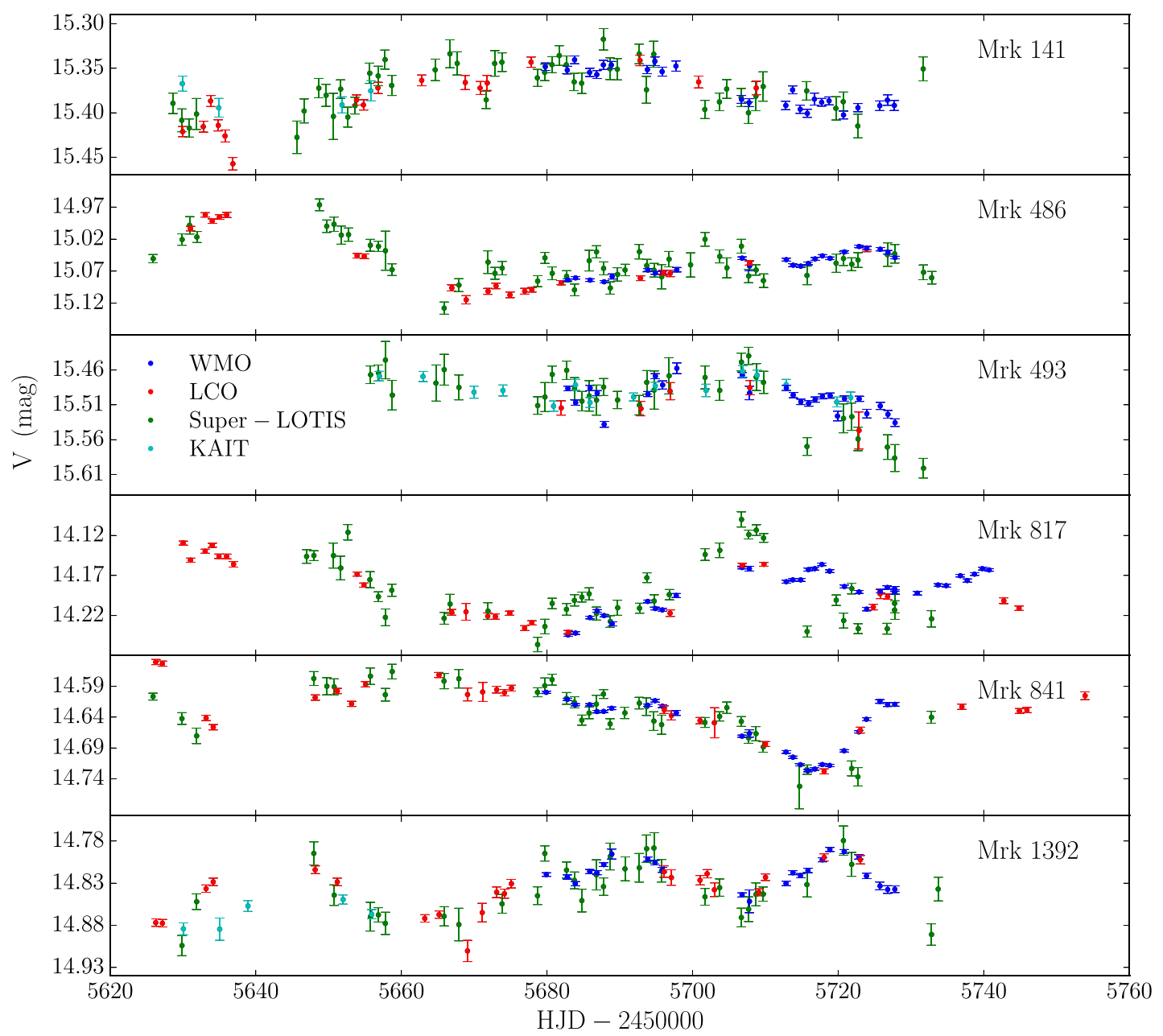

Figure 2. Same as Figure 1, but for standard aperture photometry light curves.

Table 6

$V$-band Light-curve Variability Statistics

\begin{tabular}{lcccc}
\hline \hline Object & $\begin{array}{c}\langle V\rangle \\
(\mathrm{mag})\end{array}$ & $\begin{array}{c}\sigma \\
(\mathrm{mag})\end{array}$ & $F_{\text {var }}$ & $R_{\max }$ \\
\hline Mrk 40 & 15.52 & 0.09 & 0.08 & 1.43 \\
Mrk 50 & 15.44 & 0.15 & 0.13 & 1.68 \\
Mrk 141 & 15.38 & 0.03 & 0.02 & 1.14 \\
Mrk 279 & 14.86 & 0.02 & 0.02 & 1.08 \\
Mrk 486 & 15.06 & 0.03 & 0.03 & 1.16 \\
Mrk 493 & 15.49 & 0.03 & 0.02 & 1.16 \\
Mrk 504 & 15.83 & 0.03 & 0.02 & 1.13 \\
Mrk 704 & 14.82 & 0.08 & 0.08 & 1.27 \\
Mrk 817 & 13.17 & 0.03 & 0.03 & 1.15 \\
Mrk 841 & 14.63 & 0.05 & 0.04 & 1.20 \\
Mrk 1392 & 15.62 & 0.05 & 0.05 & 1.20 \\
Mrk 1511 & 14.83 & 0.03 & 0.02 & 1.13 \\
NGC 4593 & 13.87 & 0.12 & 0.11 & 1.57 \\
PG 1310-108 & 15.55 & 0.04 & 0.04 & 1.18 \\
Zw 229-015 & 16.08 & 0.06 & 0.05 & 1.19 \\
\hline
\end{tabular}

Note. Variability characteristics include the square root of the variance of the magnitude $\sigma$, the ratio of the maximum to the minimum flux $R_{\max }$, and the normalized excess variance of the flux $F_{\text {var }}$. The mean $V$-band magnitude value $\langle V\rangle$ of the light curve for each AGN is also given. Before calculating $F_{\text {var }}$ and $R_{\max }$ the light curves are converted from magnitudes to fluxes using the Vega calibration of the Johnson system. stars that do not fall on a bad row in any of the images. The P60 data typically have the largest uncertainties and have only been included for the Mrk 50 light curve, since it has the densest sampling of any of the AGNs, ensuring that the P60 data agree with the other data. There is less agreement between P60 data and data from other telescopes in the remaining objects.

While the light curves from image subtraction for different telescopes agree very well, there is some discrepancy between telescopes for the standard aperture photometry light curves, most noticeably for Mrk 817. These light curves are not aligned using the GP model for AGN variability that is applied to the imagesubtraction light curves, so the difference may be due to variations in telescope or filter properties. Despite this issue, the standard aperture photometry procedure has two main advantages compared to the image-subtraction procedure. First, the standard aperture photometry is fast, requiring less parameter fine-tuning and inspection of images at different stages of the analysis. Second, because the light curves for different telescopes are aligned independently, they do not need to have data points that overlap, as is required for the GP model used with the image-subtraction procedure. The data set for Mrk 486 is a good example where GP alignment would not be well constrained. For these reasons, standard aperture photometry represents an ideal method for 
measuring AGN light curves that will not undergo detailed velocity-resolved analysis.

\subsection{Continuum Variability}

As expected for reverberation mapping data sets, there is a range in variability within the sample. In addition, variability can be observed on a variety of timescales, as illustrated by the long-timescale variations for Mrk 50 compared to the shorttimescale variations for NGC 4593. These differences demonstrate why a reverberation mapping campaign with high cadence over a long period of time is necessary to characterize the variability of an AGN sample.

We measure three variability characteristics for each light curve. The measurements of variability include the square root of the variance in the $V$-band magnitude $\sigma$, the ratio of the maximum to the minimum flux $R_{\max }$, and finally the normalized excess variance of the flux $F_{\text {var }}$. The values of each of these measurements, including the mean $V$-band magnitude, are given in Table 6 . The normalized excess variance as defined by Rodríguez-Pascual et al. (1997) measures the variability of the source after correcting for measurement uncertainties and normalization by the mean flux, $\langle f\rangle$ :

$$
F_{\mathrm{var}}=\frac{\sqrt{\sigma^{2}-\delta^{2}}}{\langle f\rangle},
$$

where $\sigma^{2}$ is the variance in the fluxes and $\delta^{2}$ is the mean square value of the individual flux uncertainty measurements.

While the six AGNs with standard aperture photometry light curves have variations of $\sim 0.1 \mathrm{mag}$ over the course of the campaign, the nine AGNs with image-subtraction photometry light curves have variations of $0.1-0.4$ mag. Mrk 50 and NGC 4593 show the largest change in flux, and this is reflected in their values of $F_{\text {var }}>0.1$, while the rest of the sample has values of $F_{\text {var }}$ ranging from 0.02 to 0.08 . Of course, the overall amplitude of variability is not the only important light-curve characteristic for reverberation mapping: the trend of the light curve and the clarity with which a light curve changes slope are critical. Monotonically changing light curves, as shown in the beginning of the light curve of Mrk 704, or light curves with very slow changes (e.g., for Mrk 141 and the first half of the Mrk 841 light curve), can make timelag measurements difficult.

\section{Conclusions}

We present $V$-band continuum light curves for the 15 AGNs in the LAMP 2011 reverberation mapping data set. For the AGNs with high levels of $\mathrm{H} \beta$ variability (see Barth et al. 2015), the AGN continuum light curves are measured using imagesubtraction software in order to create high-quality reverberation mapping data for velocity-resolved analysis such as dynamical modeling of the BLR (e.g., Pancoast et al. 2014; Grier et al. 2017; Pancoast et al. 2018). For the AGNs with lower levels of $\mathrm{H} \beta$ variability, the AGN continuum light curves are measured using standard aperture photometry techniques. This combination of techniques allows for more precise photometry measurements for the most variable AGNs, while taking advantage of less time-intensive analysis for those light curves less likely to provide strong reverberation mapping signatures.

These $V$-band photometry light curves will be combined with the spectra measured by Barth et al. (2015) to produce final cross-correlation lag and $\mathrm{BH}$ mass measurements (A. Barth et al., in preparation). Having independent photometry of the AGN continuum ensures that no spurious time-lag signals are introduced between the emission lines and the AGN continuum as measured from the same spectra. Independent photometry also provides a more densely sampled AGN continuum light curve that is particularly helpful for dynamical modeling of the BLR. So far, 8 of the 15 LAMP 2011 AGNs have constraints on their BLR geometry and dynamics from modeling this data set (Pancoast et al. 2018; Williams et al. 2018). Future work will include developing more physically motivated BLR models to take full advantage of these data.

We thank the Lick Observatory staff for their exceptional support during our observing campaign. The Lick AGN Monitoring Project 2011 is supported by NSF grants AST1107812, 1107865, 1108665, and 1108835. M.D.J. would like to thank the Brigham Young University College of Physical and Mathematical Sciences for continued support of the West Mountain Observatory research facilities. During the LAMP 2011 campaign, WMO received additional support from NSF grant AST-0618209. This research was made possible through the use of the AAVSO Photometric All-Sky Survey (APASS), funded by the Robert Martin Ayers Sciences Fund. Work at UC Irvine was additionally supported by NSF grant AST-1412693. The Dark Cosmology Centre (DARK) is funded by the Danish National Research Foundation. A.P. is supported by NASA through Einstein Postdoctoral Fellowship grant No. PF5160141 awarded by the Chandra X-ray Center, which is operated by the Smithsonian Astrophysical Observatory for NASA under contract NAS8-03060. A.V.F. is grateful for the financial assistance of the TABASGO Foundation, the Christopher R. Redlich Fund, and the Miller Institute for Basic Research in Science (UC Berkeley). Research by D.J.S. is supported by NSF grants AST-1821967, 1821987, 1813708, and 1813466. The work of D.S. was carried out at the Jet Propulsion Laboratory, California Institute of Technology, under a contract with NASA. J.H.W. acknowledges support by the National Research Foundation of Korea (NRF) grant funded by the Korean government (No.2017R1A5A1070354). T.T. is grateful to the Packard Foundation for support through a Packard Research Fellowship and to the NSF for support through grants AST-1107865 and AST-1412315. V.N.B. gratefully acknowledges assistance from an NSF Research at Undergraduate Institutions (RUI) grant AST-1312296. Research at Lick Observatory is partially supported by a generous gift from Google. Note that our findings and conclusions do not necessarily represent the views of the NSF.

\section{ORCID iDs}

Anna Pancoast (ib https://orcid.org/0000-0003-1065-5046 Andreas Skielboe (iD https://orcid.org/0000-0001-9765-3603 Vardha N. Bennert (iD https://orcid.org/0000-0003-2064-0518 David J. Sand (10 https://orcid.org/0000-0003-4102-380X Aaron J. Barth (i) https://orcid.org/0000-0002-3026-0562 Tommaso Treu (iD https://orcid.org/0000-0002-8460-0390 C. David Laney (i) https://orcid.org/0000-0003-2967-110X Alexei V. Filippenko (i) https://orcid.org/0000-0003-3460-0103 Matthew A. Malkan (i) https://orcid.org/0000-0001-6919-1237 Daniel Stern (iD https://orcid.org/0000-0003-2686-9241 Jong-Hak Woo (iD https://orcid.org/0000-0002-8055-5465 


\section{References}

Abazajian, K. N., Adelman-McCarthy, J. K., Agüeros, M. A., et al. 2009, ApJS, 182, 543

Alard, C. 2000, A\&AS, 144, 363

Alard, C., \& Lupton, R. H. 1998, ApJ, 503, 325

Barth, A. J., Bennert, V. N., Canalizo, G., et al. 2015, ApJS, 217, 26

Barth, A. J., Nguyen, M. L., Malkan, M. A., et al. 2011a, ApJ, 732, 121

Barth, A. J., Pancoast, A., Bennert, V. N., et al. 2013, ApJ, 769, 128

Barth, A. J., Pancoast, A., Thorman, S. J., et al. 2011b, ApJL, 743, L4

Becker, A. 2015, HOTPANTS: High Order Transform of PSF ANd Template Subtraction, Astrophysics Source Code Library, ascl:1504.004

Bentz, M. C., Horne, K., Barth, A. J., et al. 2010a, ApJL, 720, L46

Bentz, M. C., \& Katz, S. 2015, PASP, 127, 67

Bentz, M. C., Peterson, B. M., Netzer, H., Pogge, R. W., \& Vestergaard, M. 2009a, ApJ, 697, 160

Bentz, M. C., Walsh, J. L., Barth, A. J., et al. 2009b, ApJ, 705, 199

Bentz, M. C., Walsh, J. L., Barth, A. J., et al. 2010b, ApJ, 716, 993

Blandford, R. D., \& McKee, C. F. 1982, ApJ, 255, 419

Brewer, B. J., Pártay, L. B., \& Csányi, G. 2011, Stat. Comp., 21, 649

Brown, T. M., Baliber, N., Bianco, F. B., et al. 2013, PASP, 125, 1031

Cenko, S. B., Fox, D. B., Moon, D.-S., et al. 2006, PASP, 118, 1396

Denney, K. D., Peterson, B. M., Pogge, R. W., et al. 2010, ApJ, 721, 715

Doroshenko, V. T., Sergeev, S. G., Merkulova, N. I., et al. 2005a, Ap, 48,156

Doroshenko, V. T., Sergeev, S. G., Merkulova, N. I., et al. 2005b, Ap, 48, 304

Du, P., Lu, K.-X., Hu, C., et al. 2016, ApJ, 820, 27

Fausnaugh, M. M., Denney, K. D., Barth, A. J., et al. 2016, ApJ, 821, 56

Filippenko, A. V., Li, W. D., Treffers, R. R., \& Modjaz, M. 2001, in ASP Conf. Ser. 246, IAU Coll. 183: Small Telescope Astronomy on Global Scales, ed. B. Paczynski, W.-P. Chen, \& C. Lemme (San Francisco, CA: ASP), 121

Greene, J. E., Hood, C. E., Barth, A. J., et al. 2010, ApJ, 723, 409

Grier, C. J., Pancoast, A., Barth, A. J., et al. 2017, ApJ, 849, 146

Grier, C. J., Peterson, B. M., Horne, K., et al. 2013, ApJ, 764, 47
Kasliwal, V. P., Vogeley, M. S., \& Richards, G. T. 2015, MNRAS, 451, 4328

Kelly, B. C., Bechtold, J., \& Siemiginowska, A. 2009, ApJ, 698, 895

Kozłowski, S., Kochanek, C. S., Udalski, A., et al. 2010, ApJ, 708, 927

Landolt, A. U. 1992, AJ, 104, 340

Lang, D., Hogg, D. W., Mierle, K., Blanton, M., \& Roweis, S. 2010, AJ, 139, 1782

Li, Y.-R., Wang, J.-M., Hu, C., Du, P., \& Bai, J.-M. 2014, ApJL, 786, L6

MacKay, D. J. C. 2003, Information Theory, Inference and Learning Algorithms (Cambridge: Cambridge Univ. Press)

MacLeod, C. L., Ivezić, Ž, Kochanek, C. S., et al. 2010, ApJ, 721, 1014

Mushotzky, R. F., Edelson, R., Baumgartner, W., \& Gandhi, P. 2011, ApJL, 743, L12

Pancoast, A., Barth, A. J., Horne, K., et al. 2018, ApJ, 856, 108

Pancoast, A., Brewer, B. J., \& Treu, T. 2011, ApJ, 730, 139

Pancoast, A., Brewer, B. J., Treu, T., et al. 2012, ApJ, 754, 49

Pancoast, A., Brewer, B. J., Treu, T., et al. 2014, MNRAS, 445, 3073

Park, D., Woo, J.-H., Treu, T., et al. 2012, ApJ, 747, 30

Pei, L., Barth, A. J., Aldering, G. S., et al. 2014, ApJ, 795, 38

Peterson, B. M. 1993, PASP, 105, 247

Peterson, B. M., Ferrarese, L., Gilbert, K. M., et al. 2004, ApJ, 613, 682

Peterson, B. M., \& Wandel, A. 1999, ApJL, 521, L95

Rasmussen, C. E., \& Williams, C. K. I. 2006, Gaussian Processes for Machine Learning (Cambridge, MA: MIT Press)

Rodríguez-Pascual, P. M., Alloin, D., Clavel, J., et al. 1997, ApJS, 110, 9

Schlafly, E. F., \& Finkbeiner, D. P. 2011, ApJ, 737, 103

Skielboe, A., Pancoast, A., Treu, T., et al. 2015, MNRAS, 454, 144

Tonry, J. L., Schmidt, B. P., Barris, B., et al. 2003, ApJ, 594, 1

Valenti, S., Sand, D. J., Barth, A. J., et al. 2015, ApJL, 813, L36

van Dokkum, P. G. 2001, PASP, 113, 1420

Walsh, J. L., Minezaki, T., Bentz, M. C., et al. 2009, ApJS, 185, 156

Williams, P. R., Pancoast, A., Treu, T., et al. 2018, ApJ, 866, 75

Woo, J.-H., Treu, T., Barth, A. J., et al. 2010, ApJ, 716, 269

Xiao, M., Du, P., Horne, K. D., et al. 2018, ApJ, 864, 109

Zu, Y., Kochanek, C. S., Kozłowski, S., \& Udalski, A. 2013, ApJ, 765, 106 